\title{
Diagnosing Gastric Mesenchymal Tumors by Digital Endoscopic Ultrasonography Image Analysis
}

\author{
Moon Won Lee and Gwang Ha Kim \\ Department of Internal Medicine, Pusan National University School of Medicine and Biomedical Research Institute, Pusan National \\ University Hospital, Busan, Korea
}

Gastric mesenchymal tumors (GMTs) are incidentally discovered in national gastric screening programs in Korea. Endoscopic ultrasonography (EUS) is the most useful diagnostic modality for evaluating GMTs. The differentiation of gastrointestinal stromal tumors from benign mesenchymal tumors, such as schwannomas or leiomyomas, is important to ensure appropriate clinical management. However, this is difficult and operator dependent because of the subjective interpretation of EUS images. Digital image analysis computes the distribution and spatial variation of pixels using texture analysis to extract useful data, enabling the objective analysis of EUS images and decreasing interobserver and intraobserver agreement in EUS image interpretation. This review aimed to summarize the usefulness and future of digital EUS image analysis for GMTs based on published reports and our experience. Clin Endosc 2021;54:324-328

Key Words: Computer-assisted; Endosonography; Image processing; Mesenchymal tumor; Stomach

\section{INTRODUCTION}

Gastric mesenchymal tumors (GMTs) are accidentally discovered as protruding firm subepithelial lesions during upper endoscopy, particularly in national gastric cancer screening programs in Korea. ${ }^{1}$ These tumors usually appear as spindle-shaped cells and display smooth muscle or nerve sheath differentiation on histopathology. Most GMTs are gastrointestinal stromal tumors (GISTs) derived from interstitial cells of Cajal. ${ }^{1,2}$ Because GISTs have a risk of metastasis, particularly to the liver and peritoneum, even after surgery for localized diseases, ${ }^{3,4}$ all GISTs are considered potentially malignant and candidates for resection, especially when they are larger than 1

Received: March 1, 2020 Revised: April 2, 2020

Accepted: April 2, 2020

Correspondence: Gwang Ha Kim

Department of Internal Medicine, Pusan National University School of Medicine and Biomedical Research Institute, Pusan National University Hospital, 179 Gudeok-ro, Seo-gu, Busan 49241, Korea

Tel: +82-51-240-7869, Fax: +82-51-244-8180, E-mail: doc0224@pusan.ac.kr ORCID: https://orcid.org/0000-0001-9721-5734

(cc) This is an Open Access article distributed under the terms of the Creative Commons Attribution Non-Commercial License (http://creativecommons.org/ licenses/by-nc/3.0) which permits unrestricted non-commercial use, distribution, and reproduction in any medium, provided the original work is properly cited. $\mathrm{cm}^{4-6}$

Differentiating GISTs from benign mesenchymal tumors, such as schwannomas or leiomyomas, is important to ensuring proper clinical decisions. Endoscopic ultrasonography (EUS) is the most useful diagnostic modality for evaluating gastrointestinal subepithelial lesions because it enables the demonstration of margins, echogenicity, layer of origin, and detailed morphology. ${ }^{7-9}$ Although many studies have attempted to differentiate GISTs from benign GMTs using EUS, the results are controversial. ${ }^{9,10}$ Because of subjective interpretation of EUS image findings, limitations such as poor interobserver agreement persist in the analysis of the characteristic features of GMTs. ${ }^{11,12}$ To overcome these limitations, digital image analysis is expected to help endoscopists improve GMT diagnosis accuracy. Here we summarize the usefulness and future of digital EUS image analysis for GMTs based on published reports and our experience.

\section{ENDOSCOPIC ULTRASONOGRAPHY FEATURES OF GASTRIC MESENCHYMAL TUMORS}

During EUS examinations of GMTs, endoscopists should 
carefully recognize the following features: (1) tumor location; (2) presence of mucosal ulceration on endoscopy and/ or EUS; (3) maximal diameter; (4) echogenicity relative to the surrounding normal proper muscle layer (hyperechoic, isoechoic, or hypoechoic); (5) homogeneity (homogenous or heterogeneous); (6) presence of cystic spaces, hyperechogenic spots, and calcification; (7) presence of a marginal halo and lobulation; (8) regularity of the marginal border (regular or irregular); and (9) tumor growth pattern (inside or outside the gastric wall). ${ }^{13}$ Of them, several EUS features of GMTs can provide important clues to ensure the correct diagnosis and appropriate management (Fig. 1). According to our previous studies, tumor location, tumor echogenicity relative to the surrounding normal proper muscle layer, homogeneity, and presence/absence of hyperechogenic spots and marginal halo are helpful for diagnosing GMTs (Table 1).

\section{Leiomyoma}

Leiomyomas are benign tumors that originate from the muscularis mucosa or the muscularis propria of the gastrointestinal tract. Gastric leiomyomas are usually found in the cardia and upper body. On EUS, leiomyomas are well-circumscribed homogeneously hypoechoic lesions with an echogenicity that is similar to that of the surrounding proper muscle layer. Calcifications are relatively common in leiomyomas $(6.5 \%-18 \%)$ but rare in GISTs and schwannomas (0\%-3.5\% and $0 \%-3.7 \%$, respectively). ${ }^{14-16}$

\section{Schwannoma}

Schwannomas are tumors of spindle cells that arise from the benign nerve sheath of Schwann cells. Gastric schwannomas are usually found at a rate of $57 \%-81 \%$ in the body, $7 \%-40 \%$ in the antrum, and $0 \%-29 \%$ in the fundus, especially in middle-aged women. ${ }^{16-18}$ On EUS, they are heterogeneously or homogeneously hypoechoic lesions with decreased echogenicity relative to the surrounding proper muscle layer. ${ }^{16}$ Since schwannomas have a peripheral lymphoid cuff around the lesion, a prominent marginal halo is seen on EUS at a rate of $71 \%-89 \% \cdot{ }^{16-18}$ However, the marginal halo is not a unique EUS finding of schwannomas; rather, it is also frequently observed in GISTs but with a different mechanism. GISTs represent a capsule-like structure that is partially or completely circumscribed by the surrounding proper muscle. Therefore, the marginal halo of GISTs is thinner than that of schwannoma. ${ }^{9,10}$

\section{Gastrointestinal stromal tumors}

GISTs are the most commonly discovered GMTs and have malignant potential. Gastric GISTs are usually found in the
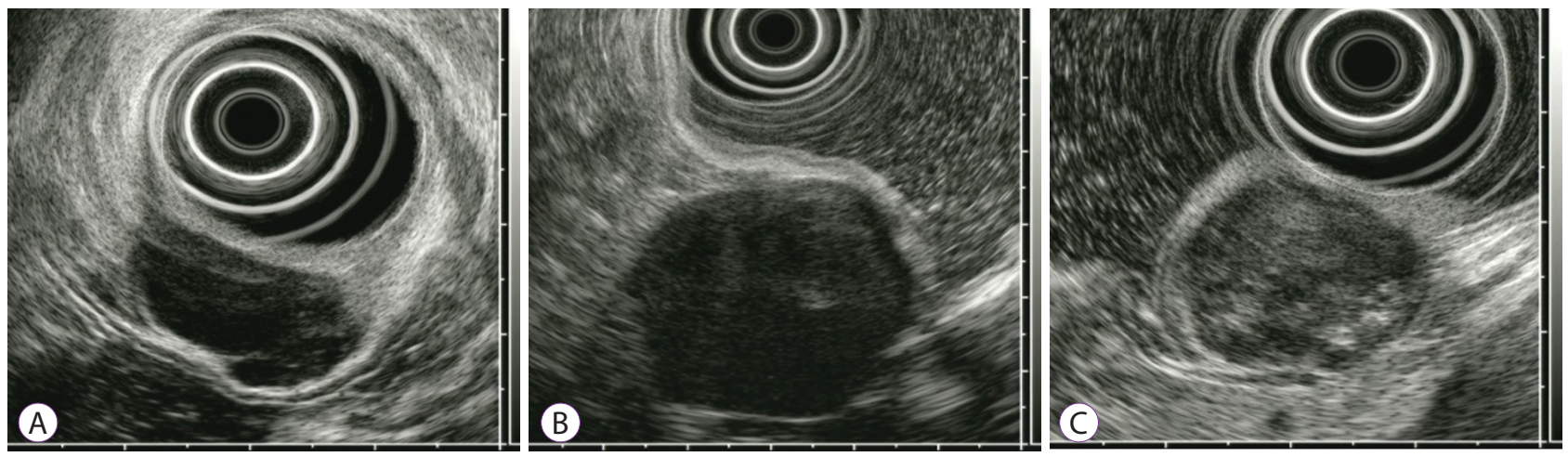

Fig. 1. Endoscopic ultrasonography features of gastric mesenchymal tumors: (A) leiomyoma; (B) schwannoma; (C) gastrointestinal mesenchymal tumor.

Table 1. Characteristic Endoscopic Ultrasonography Features of Gastric Mesenchymal Tumors

\begin{tabular}{lccc}
\hline EUS feature & Leiomyoma & Schwannoma & GIST \\
\hline Tumor location & Cardia, upper body & Body & Body, fundus \\
Homogeneity & Homogeneous & Homo/heterogeneous & Heterogeneous \\
Echogenicity compared to surrounding muscle echo & Isoechoic & Hypoechoic & Hyperechoic \\
Marginal halo & $(-)$ & $(++)$ & $(+)$ \\
Hyperechogenic foci & $(-)$ & $(+/-)$ & $(+)$ \\
\hline
\end{tabular}

EUS, endoscopic ultrasonography; GIST, gastrointestinal stromal tumor. 
body and fundus (in $46 \%-58 \%$ and $21 \%-33 \%$, respectively) but rarely found in the antrum and cardia $(13 \%-18 \%$ and $2 \%-8 \%$, respectively). ${ }^{14,19}$ Many previous studies attempted to demonstrate the ability of EUS to differentiate GISTs from other GMTs, but the results are inconsistent. On EUS, GISTs show hypoechoic and heterogeneous echo patterns with a marginal halo and hyperechoic spots. The echogenicity of GISTs is slightly higher than that of the surrounding proper muscle layer. ${ }^{9}$ Several EUS features such as size, irregular margin, cystic change, presence of hyperechogenic foci, and peritumoral lymphadenopathy are suggested as factors predictive of a high risk of malignant potential; ${ }^{8,20}$ according to our previous study, only tumor size $(>3.5 \mathrm{~cm})$ is the most accurate factor for predicting malignancy.

\section{DIGITAL ENDOSCOPIC}

\section{ULTRASONOGRAPHY IMAGE ANALYSIS FOR GASTRIC MESENCHYMAL TUMORS}

We have previously reported that hyperechogenic spots, a peripheral halo, heterogeneity, and hyperechogenicity in comparison with the surrounding proper muscle layer are important for differentiating GISTs from other GMTs. ${ }^{9}$ There is high sensitivity $(89.1 \%)$ and specificity $(85.7 \%)$ in the presence of at least two of these four features for predicting GISTs. However, as abovementioned, the interpretation of EUS images is subjective, which can result in poor interobserver agreement. To overcome this limitation, we must objectively analyze the EUS images of GMTs.

However, EUS images display different characteristics according to actual EUS settings such as gain and contrast, different echoendoscopes (mechanical vs. electronic), and EUS systems used during EUS examinations. Thus, the standardization of EUS images is required to minimize these differences. Accordingly, in our previous studies, we selected the least variable portion of the EUS images such as the outer hyperechoic rim and anechoic center of the echoendoscope and processed the standardization. ${ }^{21,22}$ Next, we attempted to find a method to objectively evaluate EUS findings such as homogeneity and echogenicity grades. EUS images are pixels that compose black and white images, and the brightness value (range, 0-255) represents their echo density. Therefore, we thought that analysis of the brightness values can be an appropriate method to evaluate echogenicity level and heterogeneity degree. As a result, the echogenicity level and heterogeneity degree were expressed as mean $\left(\mathrm{T}_{\text {mean }}\right)$ and standard deviation $\left(\mathrm{T}_{\mathrm{SD}}\right)$ of the brightness values. Based on the above processes, we developed a diagnostic system using the digital EUS image analysis of GMTs (Fig. 2).

In our first study of 65 GMTs, the $\mathrm{T}_{\text {mean }}$ and $\mathrm{T}_{\mathrm{SD}}$ were significantly higher in GISTs than in leiomyomas and schwanno-

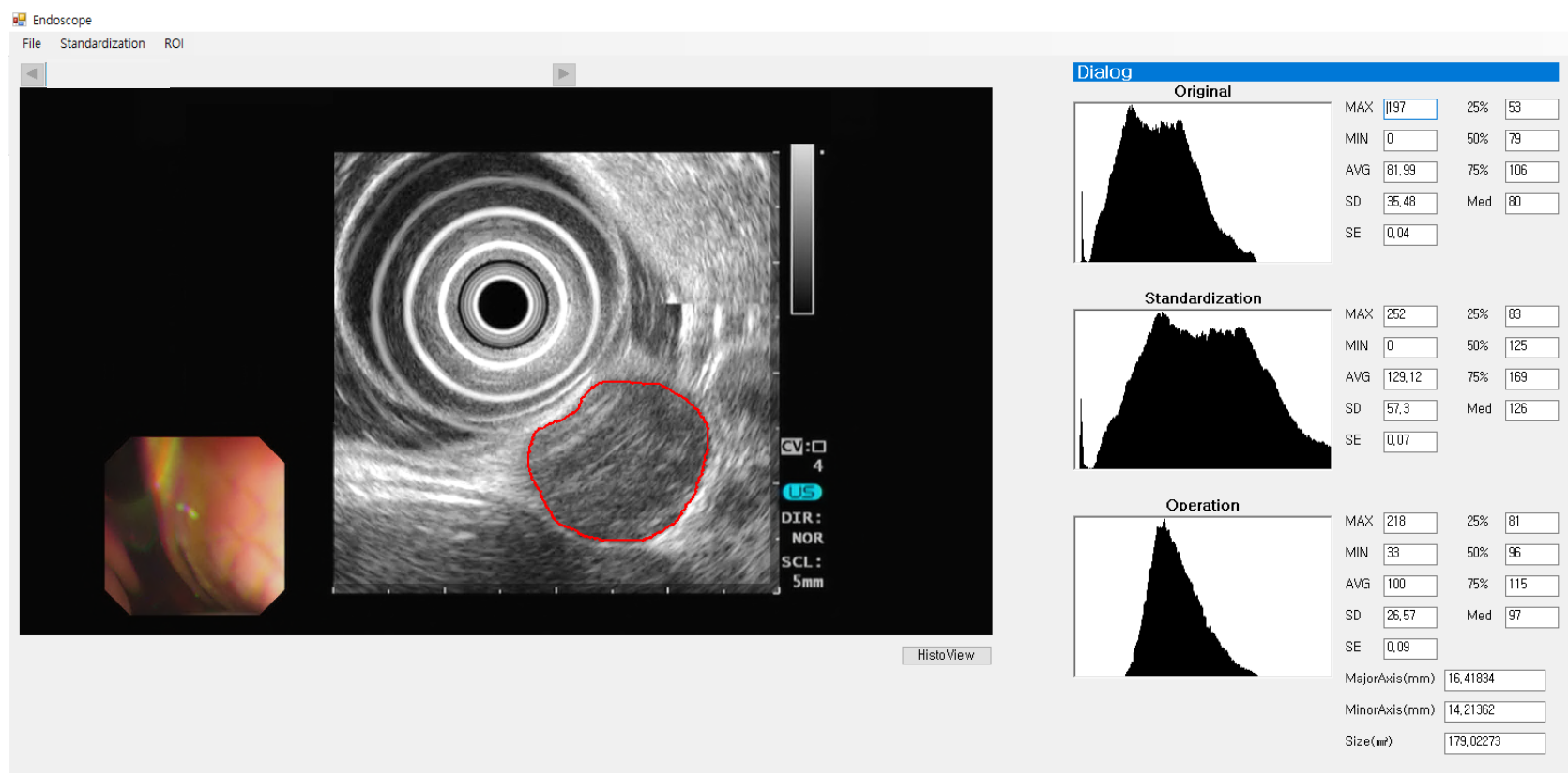

Fig. 2. Example of digital endoscopic ultrasonography image analysis of a gastric mesenchymal tumor. From the standardized image, a region of interest (ROI) is selected by an endoscopist for tumor analysis. The results for the ROI are expressed in the bottom histogram. The mean and standard deviation of the brightness values are 96 and 26.57 , respectively. 
mas. ${ }^{22}$ When a receiver operating characteristic (ROC) curve was created to identify the best sensitivity and specificity cutoff values of $\mathrm{T}_{\text {mean }}$ and $\mathrm{T}_{\mathrm{SD}}$ for differentiating GISTs from leiomyomas or schwannomas, the sensitivity and specificity was almost optimized when $\mathrm{T}_{\text {mean }}$ was $\geq 65$ and $\mathrm{T}_{\mathrm{SD}}$ was $\geq 75$ for predicting GISTs. There was high sensitivity (94\%) and specificity $(80 \%)$ in the presence of at least one of these two findings for predicting GISTs.

Next, we focused on GMTs measuring 2-5 cm. According to recent guidelines for gastric subepithelial tumors, when GMTs are smaller than $2 \mathrm{~cm}$, they can usually be followed by periodic endoscopy or EUS once or twice a year until the tumors increase in size or become symptomatic, even if they are diagnosed as GISTs later. ${ }^{13,21}$ However, surgical resection is recommended for GMTs measuring $>5 \mathrm{~cm}$. If a GMT measures $2-5 \mathrm{~cm}$, the clinical decision process can be shared with patients regarding whether to perform a histopathological diagnosis examination (for example, by EUS-guided fine-needle aspiration/biopsy or by deep biopsy via endoscopic submucosal dissection) or whether the patient requires surgical resection. Therefore, we tried to develop a scoring system to predict GISTs in 103 GMTs measuring $2-5 \mathrm{~cm}$ using digital EUS image analysis. ${ }^{21}$ Similar to our previous study, $\mathrm{T}_{\text {mean }}$ and $\mathrm{T}_{\mathrm{SD}}$ were significantly higher in GISTs than in non-GIST tumors. In addition, patients with GISTs were older than those with nonGIST tumors. When ROC curves were created, the sensitivity and specificity were almost optimized for differentiating GISTs from non-GIST tumors when the critical values of age, $T_{\text {mean }}$, and $\mathrm{T}_{\mathrm{SD}}$ were 57.5 years, 67.0 , and 25.6, respectively. Based on

Table 2. Gastrointestinal Stromal Tumor Predicting Scoring System for Gastric Mesenchymal Tumors

\begin{tabular}{lcc}
\hline \multirow{2}{*}{ Variables } & \multicolumn{2}{c}{ Points } \\
\cline { 2 - 3 } & $(+)$ & $(-)$ \\
\hline Age $\geq 58$ yr & 2 & 0 \\
$\mathrm{~T}_{\text {mean }} \geq 67$ & 3 & 0 \\
$\mathrm{~T}_{\mathrm{SD}} \geq 26$ & 1 & 0
\end{tabular}

Adapted from the article of Lee et al. Gastric Cancer 2019;22:980$987^{21}$ the $\beta$-coefficient values of multivariate analysis, we created a GIST predicting scoring by assigning 3 points for $\mathrm{T}_{\text {mean }} \geq 67,2$ points for age $\geq 58$ years, and 1 point for $\mathrm{T}_{\mathrm{SD}} \geq 26$ (Table 2 ).

GMTs with 3 or more points predicted GISTs with a sensitivity of $86.5 \%$ (95\% confidence interval [CI], 80.3\%-91.0\%), specificity of $75.9 \%$ ( $95 \% \mathrm{CI}, 60.0 \%-87.4 \%$ ), and accuracy of 83.5\% (95\% CI, 74.6\%-90.0\%). Considering the diagnostic yield of EUS-guided fine-needle aspiration/biopsy for subepithelial tumors is $60 \%-85 \%,{ }^{23-25}$ the GIST predicting scoring system can to be useful for ensuring appropriate clinical decision. In another study using an artificial neural network based on the multilayer perceptron architecture on EUS images of gastric subepithelial tumors, the authors showed high accuracy for the differential diagnosis of malignant subepithelial tumors (GISTs and carcinoid tumors) from lipomas. ${ }^{26}$ The model was reported as "good" for the differentiation of carcinoid tumors and GISTs and "excellent" for the differentiation of lipomas, with areas under the ROC curve of $0.86,0.89$, and 0.92 , respectively.

Studies published to date on digital EUS image analysis for GMTs are summarized in Table 3. However, these studies are subjected to several limitations. First, all three studies are based on single-center experiences. As aforementioned, EUS images vary according to different clinical settings such as contrast, gain, and differences in EUS systems and echoendoscopes. Even standardization process cannot overcome these differences completely. Therefore, the results of previous studies require validation in various clinical settings. Second, because all published studies were retrospective, bias in the EUS image review process might have been unavoidable. In our studies, we selected the EUS images with the highest quality to perform the digital image analysis. However, during EUS examination, at least 10 EUS images were usually taken to determine the characteristics of GMTs; this would help compensate for the limitations of retrospective research. Accordingly, we plan to conduct a large-scale, multi-center prospective study to validate the digital EUS image analysis system used to predict the histopathology of GMTs.

Table 3. Summary of Published Studies on Digital Endoscopic Ultrasonography Image Analysis for Gastric Mesenchymal Tumors

\begin{tabular}{lcl}
\hline Study & Algorithm & \multicolumn{1}{c}{ Application } \\
\hline Nguyen et al. $(2010)^{26}$ & ANN & Classifying lipoma, GIST, and carcinoid tumor \\
Kim et al. (2014) & Hand craft & Standardization and EUS image pixel analysis for GIST, leiomyoma, and schwannoma \\
Lee et al. (2019) & Hand craft & $\begin{array}{c}\text { Standardization and scoring system for predicting GIST and non-GIST tumors (leiomyoma } \\
\text { and schwannoma) }\end{array}$ \\
\hline
\end{tabular}

ANN, artificial neural network; EUS, endoscopic ultrasonography; GIST, gastrointestinal stromal tumor. 


\section{CONCLUSIONS}

EUS provides useful information for the differential diagnosis of GMTs. Furthermore, digital EUS image analysis can provide additional help to endoscopists by decreasing interobserver variability and increasing diagnostic accuracy by enabling the objective analysis of EUS images. Future digital EUS image analysis systems will be embedded in the EUS system to enable real-time analysis. It is true that this system helps endoscopists make clinical decisions and make the differential diagnosis in patients with GMTs. However, before being used in real practice, these technological advances will require validation in prospective multicenter studies.

\section{Conflicts of Interest}

Gwang Ha Kim has been an Deputy Editor member of Clinical Endoscopy; however, he was not involved in the peer reviewer selection, evaluation, or decision process of this article. The author has no potential conflicts of interest.

Funding

This work was supported by a National Research Foundation of Korea (NRF) grant funded by the Korean government (MIST) (no. 2019R1F1A1063530).

\section{ORCID}

Moon Won Lee:

Gwang Ha Kim:

https://orcid.org/0000-0002-8411-6398 https://orcid.org/0000-0001-9721-5734

\section{REFERENCES}

1. Pidhorecky I, Cheney RT, Kraybill WG, Gibbs JF. Gastrointestinal stromal tumors: current diagnosis, biologic behavior, and management. Ann Surg Oncol 2000;7:705-712.

2. Miettinen M, Sobin LH, Sarlomo-Rikala M. Immunohistochemical spectrum of GISTs at different sites and their differential diagnosis with a reference to CD117 (KIT). Mod Pathol 2000;13:1134-1142.

3. Miettinen M, Sobin LH, Lasota J. Gastrointestinal stromal tumors of the stomach: a clinicopathologic, immunohistochemical, and molecular genetic study of 1765 cases with long-term follow-up. Am J Surg Pathol 2005;29:52-68.

4. Blay JY, Bonvalot S, Casali P, et al. Consensus meeting for the management of gastrointestinal stromal tumors. Report of the GIST consensus conference of 20-21 March 2004, under the auspices of ESMO. Ann Oncol 2005; 16:566-578.

5. Fletcher CD, Berman JJ, Corless C, et al. Diagnosis of gastrointestinal stromal tumors: a consensus approach. Int J Surg Pathol 2002;10:81-89.

6. Demetri GD, von Mehren M, Antonescu CR, et al. NCCN task force report: update on the management of patients with gastrointestinal stromal tumors. J Natl Compr Canc Netw 2010;8(Suppl 2):S1-S41; quiz S42-S44.

7. Chak A, Canto MI, Rösch T, et al. Endosonographic differentiation of benign and malignant stromal cell tumors. Gastrointest Endosc
1997;45:468-473.

8. Palazzo L, Landi B, Cellier C, Cuillerier E, Roseau G, Barbier JP. Endosonographic features predictive of benign and malignant gastrointestinal stromal cell tumours. Gut 2000;46:88-92.

9. Kim GH, Park DY, Kim S, et al. Is it possible to differentiate gastric GISTs from gastric leiomyomas by EUS? World J Gastroenterol 2009;15:3376-3381.

10. Okai T, Minamoto T, Ohtsubo K, et al. Endosonographic evaluation of c-kit-positive gastrointestinal stromal tumor. Abdom Imaging 2003;28:301-307.

11. Catalano MF, Sivak MV Jr, Bedford RA, et al. Observer variation and reproducibility of endoscopic ultrasonography. Gastrointest Endosc 1995;41:115-120

12. Gress F, Schmitt C, Savides T, et al. Interobserver agreement for EUS in the evaluation and diagnosis of submucosal masses. Gastrointest Endosc 2001;53:71-76

13. Nishida T, Blay JY, Hirota S, Kitagawa Y, Kang YK. The standard diagnosis, treatment, and follow-up of gastrointestinal stromal tumors based on guidelines. Gastric Cancer 2016;19:3-14.

14. Min YW, Park HN, Min BH, Choi D, Kim KM, Kim S. Preoperative predictive factors for gastrointestinal stromal tumors: analysis of 375 surgically resected gastric subepithelial tumors. J Gastrointest Surg 2015;19:631-638

15. Yamada Y, Kida M, Sakaguchi T, et al. A study on myogenic tumors of the upper gastrointestinal tract by endoscopic ultrasonography-with special reference to the differential diagnosis of benign and malignant lesions. Dig Endosc 1992;4:396-408.

16. Yoon JM, Kim GH, Park DY, et al. Endosonographic features of gastric schwannoma: a single center experience. Clin Endosc 2016;49:548-554.

17. Tao K, Chang W, Zhao E, et al. Clinicopathologic features of gastric schwannoma: 8-year experience at a single institution in China. Medicine (Baltimore) 2015;94:e1970.

18. Park HC, Son DJ, Oh HH, et al. Endoscopic ultrasonographic characteristics of gastric schwannoma distinguished from gastrointestinal stromal tumor. Korean J Gastroenterol 2015;65:21-26.

19. Seo SW, Hong SJ, Han JP, et al. Accuracy of a scoring system for the differential diagnosis of common gastric subepithelial tumors based on endoscopic ultrasonography. J Dig Dis 2013;14:647-653.

20. Shah P, Gao F, Edmundowicz SA, Azar RR, Early DS. Predicting malignant potential of gastrointestinal stromal tumors using endoscopic ultrasound. Dig Dis Sci 2009;54:1265-1269.

21. Lee MW, Kim GH, Kim KB, et al. Digital image analysis-based scoring system for endoscopic ultrasonography is useful in predicting gastrointestinal stromal tumors. Gastric Cancer 2019;22:980-987.

22. Kim GH, Kim KB, Lee SH, et al. Digital image analysis of endoscopic ultrasonography is helpful in diagnosing gastric mesenchymal tumors. BMC Gastroenterol 2014;14:7.

23. Kim GH, Cho YK, Kim EY, et al. Comparison of 22-gauge aspiration needle with 22-gauge biopsy needle in endoscopic ultrasonography-guided subepithelial tumor sampling. Scand J Gastroenterol 2014;49:347-354.

24. Han JP, Lee TH, Hong SJ, et al. EUS-guided FNA and FNB after onsite cytological evaluation in gastric subepithelial tumors. J Dig Dis 2016;17:582-587.

25. Zhang XC, Li QL, Yu YF, et al. Diagnostic efficacy of endoscopic ultrasound-guided needle sampling for upper gastrointestinal subepithelial lesions: a meta-analysis. Surg Endosc 2016;30:2431-2441.

26. Nguyen VX, Nguyen CC, Li B, Das A. Digital image analysis is a useful adjunct to endoscopic ultrasonographic diagnosis of subepithelial lesions of the gastrointestinal tract. J Ultrasound Med 2010;29:1345-1351. 